\title{
Early post-contrast T1 mapping yields maximal discriminatory capacity for detection of cardiac amyloid - influence of temporal T1 differences on MOLLI imaging
}

\author{
Mitchell A Cooper ${ }^{3,1^{*}}$, Thanh Nguyen ${ }^{1}$, Mathew Maurer ${ }^{5}$, Heather Landau ${ }^{4}$, Jiwon Kim ${ }^{6}$, Sattar Gojraty ${ }^{2}$, \\ Silvina P Dutruel ${ }^{1}$, Martin Prince', Yi Wang ${ }^{1,3}$, Jonathan W Weinsaft ${ }^{2}$
}

From 17th Annual SCMR Scientific Sessions

New Orleans, LA, USA. 16-19 January 2014

\section{Background}

Myocardial T1 mapping is increasingly used to diagnose and quantify disease burden in patients with known or suspected cardiac amyloid. Optimal timing for diagnostic application of T1 mapping is not established.

\section{Methods}

Myocardial T1 mapping was performed in two cohorts (1) "amyloid +" subjects, defined by biopsy-proven systemic amyloid with associated remodeling suggestive of cardiac involvement (left ventricular [LV] hypertrophy and/or atrial dilation); (2) normative controls without risk factors for amyloid or cardiovascular disease. CMR (1.5T) included 2 components - cine-CMR (SSFP) for cardiac structure/function, and T1 mapping for myocardial tissue characterization. T1 mapping was performed using a conventional modified look locker inversion recovery (MOLLI) sequence (flip angle $=30^{\circ}$; matrix $256 \times 128$; parallel imaging reduction factor $=1.5$; linear view ordering; 6 Kaiser-Bessel ramp preparation; 17 heart beat acquisition), with $\mathrm{T} 1$ calculated using an established formula ( $\mathrm{T} 1=\mathrm{T} 1 *(\mathrm{~B} / \mathrm{A}-1), \mathrm{T} 1 * \mathrm{~A}$, and $\mathrm{B}$ obtained via three-parameter exponential fit). To test time dependent differences in myocardial T1, MOLLI was acquired at sequential time points $(3,5,10,14$, 20 minutes) following intravenous administration of gadolinium $(0.2 \mathrm{mmol} / \mathrm{kg})$.

\section{Results}

10 subjects ( 5 amyloid, 5 controls) were studied ( $44 \pm 21$ years, $40 \%$ male); all amyloid affected subjects had biopsyconfirmed disease (4 light chain type, 1 transthyretin). Amyloid subjects had higher LV mass $(200 \pm 34$ vs. $101 \pm$ $34 \mathrm{gm}, \mathrm{p}=0.004)$, lower myocardial contraction fraction ( $32 \pm 8$ vs. $88 \pm 27, \mathrm{p}=0.002$ ), and larger left atrial area ( $26 \pm 5$ vs. $18 \pm 5 \mathrm{~cm} 2, \mathrm{p}=0.03$ ) than controls, but similar $\mathrm{LV}$ end-diastolic volume $(120 \pm 24$ vs. $133 \pm 45 \mathrm{ml}, \mathrm{p}=$ $0.61)$, stroke volume ( $66 \pm 10$ vs. $86 \pm 30 \mathrm{ml}, \mathrm{p}=0.20)$, and LVEF ( $56 \pm 10$ vs. $65 \pm 4 \%, \mathrm{p}=0.11)$. MOLLI was successfully acquired in all subjects at each time point: $\mathrm{T} 1$ differed significantly (all $\mathrm{p} \leq 0.05$ ) between amyloid and control groups at all times (Figure 1). However, magnitude of difference temporally decreased following gadolinium administration (Figure 2): T1 differences between patients and controls were maximal at 3 minutes post-contrast $(135 \pm 15$ vs. $310 \pm 61 \mathrm{msec}, \mathrm{p}=0.004)$ with progressive decrements thereafter, as evidenced by $57 \%$ relative difference between groups at 3 minutes and only a 36\% difference at 20 minutes following gadolinium infusion.

\section{Conclusions}

MOLLI-quantified myocardial T1 yields maximal difference between amyloid-affected subjects and normative controls within 3 minutes following gadolinium administration. Increased magnitude of T1 difference early post

${ }_{3}^{3}$ Biomedical Engineering, Cornell University, New York, New York, USA Full list of author information is available at the end of the article 


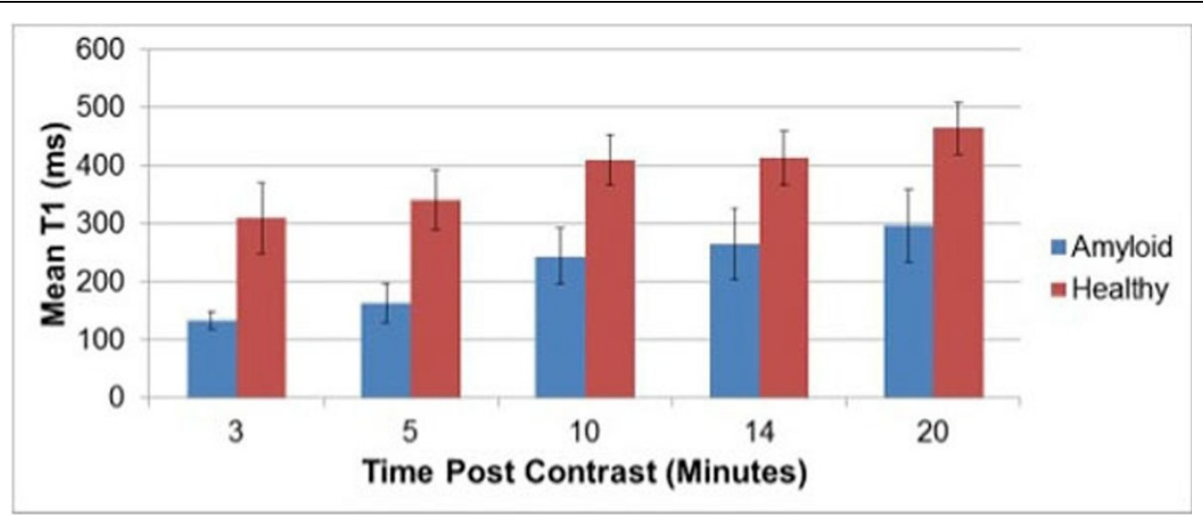

Figure 1 Mean +/- standard deviation T1 times for healthy controls and amyloid patients.

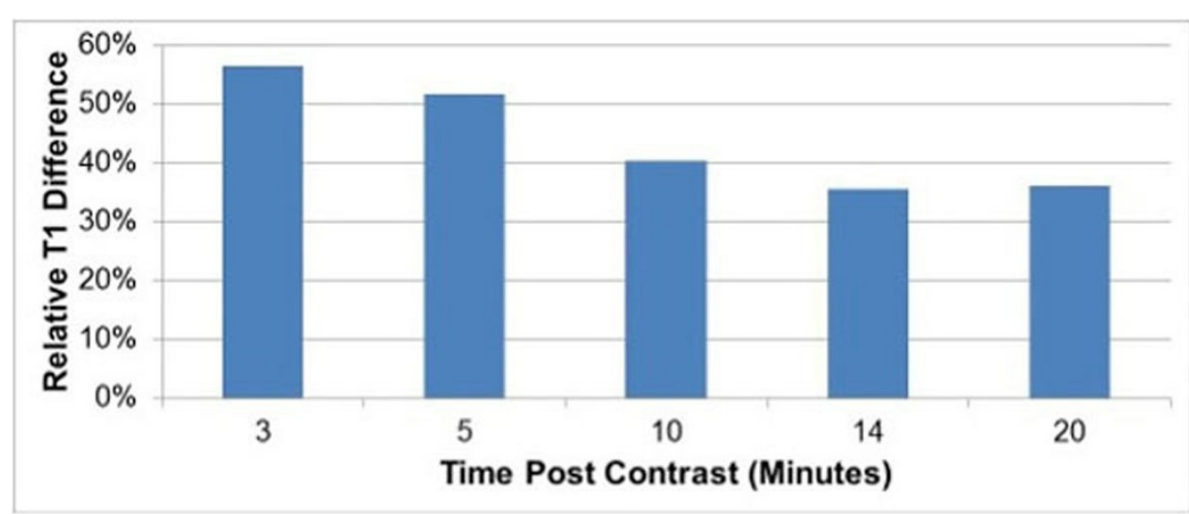

Figure 2 Percentage $\mathrm{T} 1$ difference relative to the healthy mean $\mathrm{T} 1$ time.

contrast may relate to altered gadolinium contrast kinetics due to amyloid-associated increases in cardiac extracellular volume, and/or pulse-sequence aspects of the MOLLI approach. Current findings support use of early post-contrast MOLLI T1 mapping for identification of cardiac amyloid.

\section{Funding}

M. Cooper was supported by NSF GFRP DGE-0707428. J. Weinsaft was supported by NIH K23HL102249-01.

\section{Authors' details}

${ }^{1}$ Radiology, Weill Cornell Medical College, New York, New York, USA.

${ }^{2}$ Cardiology, Weill Cornell Medical College, New York, New York, USA.

${ }^{3}$ Biomedical Engineering, Cornell University, New York, New York, USA.

${ }^{4}$ Oncology, Memorial Sloan Kettering Cancer Center, New York, New York,

USA. ${ }^{5}$ Cardiology, Columbia University Medical Center, New York, New York,

USA. ${ }^{6}$ Cardiology, Memorial Sloan Kettering Cancer Center, New York, New

York, USA.

Published: 16 January 2014
doi:10.1186/1532-429X-16-S1-P322

Cite this article as: Cooper et al:: Early post-contrast T1 mapping yields maximal discriminatory capacity for detection of cardiac amyloid influence of temporal T1 differences on MOLLI imaging. Journal of Cardiovascular Magnetic Resonance 2014 16(Suppl 1):P322.
Submit your next manuscript to BioMed Central and take full advantage of:

- Convenient online submission

- Thorough peer review

- No space constraints or color figure charges

- Immediate publication on acceptance

- Inclusion in PubMed, CAS, Scopus and Google Scholar

- Research which is freely available for redistribution

Submit your manuscript at www.biomedcentral.com/submit 